\title{
Width of the Retrocalcaneal Bursa Is Not Altered by the Ankle Motion or Flexor Hallucis Longus Contraction
}

\author{
Misako Hamada ${ }^{1, *, \dagger}$, Minori Ota ${ }^{1, \dagger}$, Nobuhide Azuma $^{2, \dagger}$ and Kotaro Yamakado ${ }^{3, \uparrow}$ \\ 1 Department of Rehabilitation, Fukui General Hospital Clinic, Fukui 910-0067, Japan; fgh-hisyo@mopera.net \\ Department of Rehabilitation, Fukui College of Health Sciences, Fukui 910-3113, Japan; fgh-ma@f-gh.jp \\ 3 Department of Orthopaedic Surgery, Fukui General Hospital, Fukui 910-8561, Japan; \\ yamakadok@gmail.com \\ * Correspondence: bb07hm@yahoo.co.jp; Tel.:+81-776-591-300; Fax: +81-776-592-538 \\ + These authors contributed equally to this work.
}

Academic Editor: Giuseppe Musumeci

Received: 19 August 2016; Accepted: 17 October 2016; Published: 1 November 2016

\begin{abstract}
The purpose of this study was to assess changes in the thickness of the retrocalcaneal bursa (RB) with respect to the ankle position with or without flexor hallucis longus (FHL) contraction. Forty feet of 20 female volunteers were examined. RB was sonographically scanned as the horizontal short-axis image of the posteromedial talar process and the calcaneal tuberosity. RB thickness was measured at three different ankle positions ( $20^{\circ}$ dorsiflexion, neutral, and $40^{\circ}$ plantar flexion) with or without FHL contraction. Without FHL contraction, the mean thickness of the RB was $2.6 \mathrm{~mm}$ in dorsiflexion, $2.4 \mathrm{~mm}$ in neutral, and $2.3 \mathrm{~mm}$ in plantar flexion. With FHL contraction, the mean thickness of the RB was $2.5 \mathrm{~mm}$ in dorsiflexion, $2.5 \mathrm{~mm}$ in neutral, and $2.3 \mathrm{~mm}$ in plantar flexion. No significant differences were detected with respect to the ankle position or FHL contraction. This study showed that in healthy subjects, the thickness of the RB in the horizontal plane did not change with respect to the ankle position with or without FHL contraction.
\end{abstract}

Keywords: retrocalcaneal bursa; ultrasound imaging; morphology

\section{Introduction}

Achilles tendon bursitis is a common cause of heel pain. The Achilles tendon bursa consists of a subcutaneous and retrocalcaneal bursa (RB) (Figure 1). The RB is located between Kager's fat pad (KFP) and the Achilles tendon, and it moves with the KFP during ankle motion (plantar flexion and dorsiflexion) [1].

RB has three functions: it acts as a shock absorber and a lubricator and removes debris from the posterior side of the Achilles tendon [2-6].

On the sagittal plane, the RB is compressed by ankle dorsiflexion, leading to impingement between the calcaneal tuberosity and the Achilles tendon [2,3]. Flexor hallucis longus (FHL) contraction facilitates the invagination of the KFP to the RB [2]. This type of compressive force is thought to be a cause of bursitis [2]. However, in the horizontal plane, the kinetics of the RB and the influence of FHL contraction are not well examined. The purpose of this study was to assess the shape (thickness) of the RB in the horizontal plane with respect to ankle position with or without FHL contraction. Our hypothesis was that the thickness of the RB in the horizontal plane changes with respect to the ankle position with or without FHL contraction. 


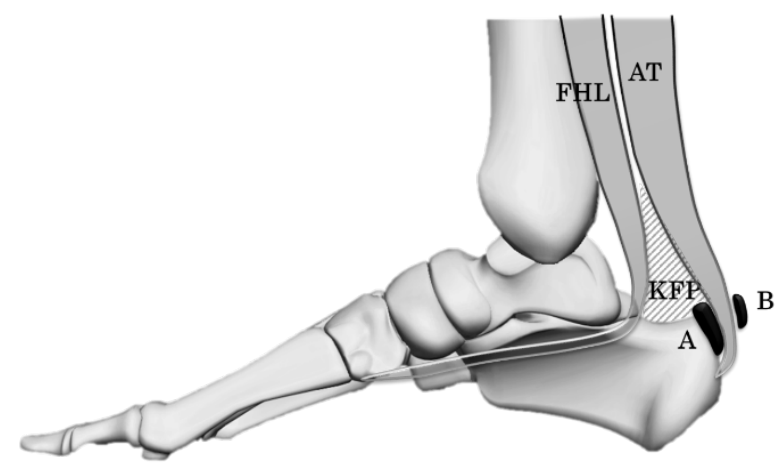

Figure 1. Retrocalcaneal bursa (A) lies in the space between the calcaneal tuberosity and Achilles tendon. Subcutaneous bursa (B) lies in the subcutaneous layer superficial to the Achilles tendon. AT, Achilles tendon; FHL, flexor hallucis longus; KFP, Kager's fat pad.

\section{Materials and Methods}

Forty feet of 20 healthy female volunteers (mean, 24.3 years; SD, 4.3 years) with the occupation of physiotherapist were examined (mean, BMI 20.3; SD, 1.5). None of the subjects had a history of significant injury around the ankle.

The thickness of RB was measured using an ultrasound imaging system (Prosound $\alpha-7$, Hitachi, Japan) in three ankle positions ( $20^{\circ}$ dorsiflexion, neutral, and $40^{\circ}$ plantar flexion) with or without FHL contraction; all subjects were kneeling $\left(90^{\circ}\right.$ hip flexion, $90^{\circ}$ knee flexion) to relax the gastrocnemius (Figure 2). A $14 \mathrm{MHz}$ linear array probe was used to measure the thickness of RB. The thickness of $\mathrm{RB}$ in the horizontal plane was defined as the distance between the posteromedial talar process and the calcaneal tuberosity (Figure 3). Before the study, the reliability of RB thickness measurement was calculated using the intraclass correlation coefficient, which ranged from 0.82 to 0.95 in each condition (ankle position and FHL contraction). The data were analyzed using the non-repeating two-way factorial analysis of variance and Tukey's method for the post-hoc test. $p \leq 0.05$ was considered as statistically significant.
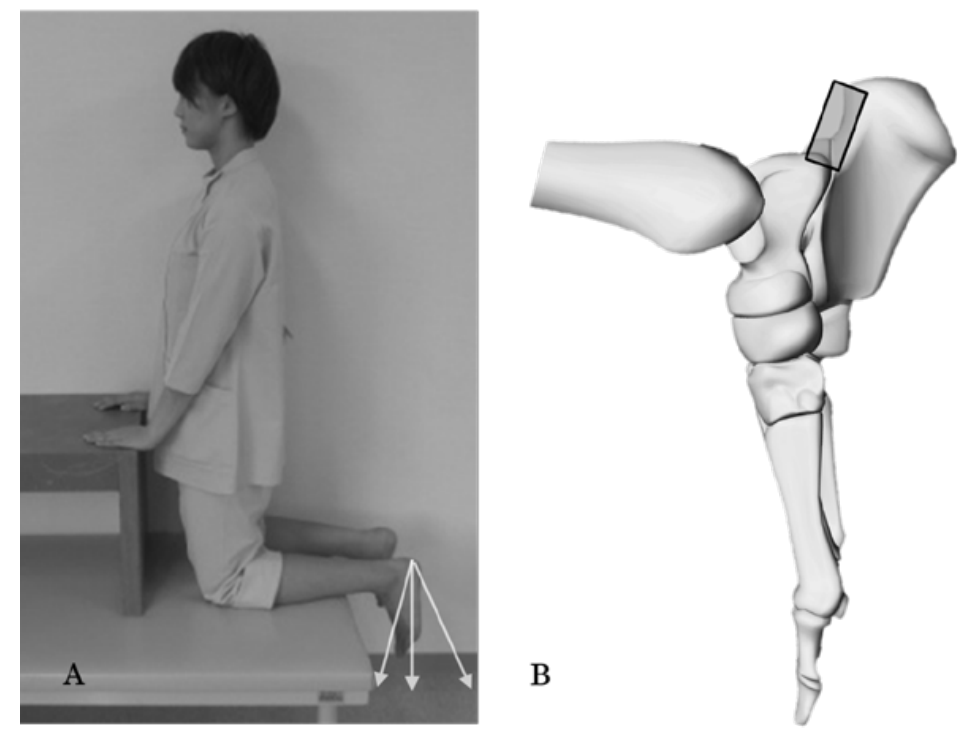

Figure 2. Probe position. Subject is placed in kneeling position (A). The probe is held from the lateral side (as shown in (B)) on the transverse plain, connecting the posteromedial talar process and the calcaneal tuberosity. Retrocalcaneal bursal thickness is measured at three different ankle positions $\left(20^{\circ}\right.$ dorsiflexion, neutral, and $40^{\circ}$ plantar flexion; arrows in (A)) with or without FHL contraction. 


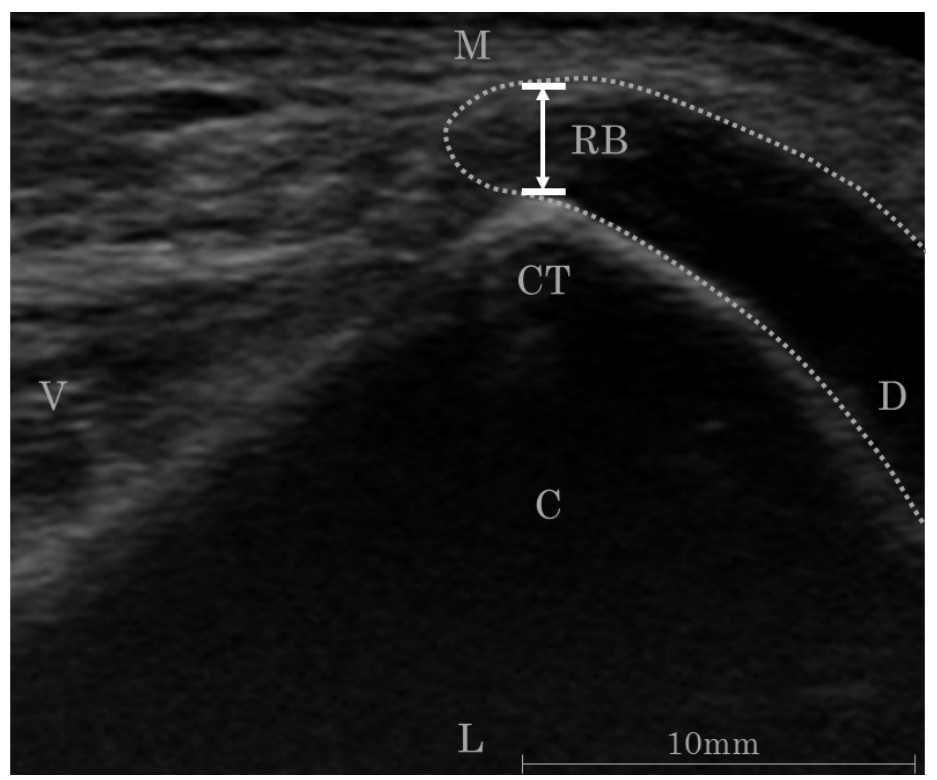

Figure 3. Ultrasound measurement of the retrocalcaneal bursal thickness. Thickness of the retrocalcaneal bursa (defined as the shortest distance from the calcaneal tuberosity to the medial border of the bursal wall) is measured on the transverse plain. RB, retrocalcaneal bursa; $\mathrm{C}$, calcaneus; $\mathrm{CT}$, calcaneal tuberosity; D, dorsal; M, medial; L, lateral; V, ventral.

\section{Results}

Without FHL contraction, the mean thickness of the RB was $2.6 \mathrm{~mm}$ in dorsiflexion, $2.4 \mathrm{~mm}$ in neutral, and $2.3 \mathrm{~mm}$ in plantar flexion (Table 1). With FHL contraction, the mean thickness of the RB was $2.5 \mathrm{~mm}$ in dorsiflexion, $2.5 \mathrm{~mm}$ in neutral, and $2.3 \mathrm{~mm}$ in plantar flexion. No significant differences were detected with respect to either ankle position or FHL contraction.

Table 1. The thickness of retrocalcaneal bursa.

\begin{tabular}{cccc}
\hline \multirow{2}{*}{ Condition of FHL } & \multicolumn{3}{c}{ Ankle Position } \\
\cline { 2 - 4 } & $\mathbf{2 0}^{\circ}$ Dorsiflexion & Netural & $\mathbf{4 0}^{\circ}$ Plantar Flexion \\
\hline Resting (without FHL contraction) & $2.6 \pm 0.8 \mathrm{~mm}$ & $2.4 \pm 0.9 \mathrm{~mm}$ & $2.3 \pm 0.9 \mathrm{~mm}$ \\
With FHL contraction & $2.5 \pm 0.8 \mathrm{~mm}$ & $2.5 \pm 0.7 \mathrm{~mm}$ & $2.3 \pm 0.8 \mathrm{~mm}$ \\
\hline
\end{tabular}

Data are expressed as means \pm standard deviation; FHL: flexor hallucis longus.

\section{Discussion}

In healthy subjects, the thickness of the RB in the horizontal plane did not change significantly with respect to the ankle position with or without FHL contraction. These results could be interpreted as the subtle change in the horizontal thickness of the RB being related to a pathological condition in the Kager triangle and it could be measured with the ultrasound easily, accurately and dynamically.

In the Kager triangle, the RB moves with KFP [2,7]. The KFP consist of three parts: a superficial Achilles tendon-associated part, a deep FHL-associated part, and distal calcaneal bursal wedge [2]. On the sagittal plane, during ankle plantar flexion, the calcaneal wedge pushes into the RB [2], and the angle between the Achilles tendon and the calcaneus widens [2]. FHL contraction facilitates the invagination of the KFP to the RB; however, our study showed a consistent thickness of the RB during ankle motion in the horizontal plane. In addition to the role of shock absorber, the RB acts as a lubricator and removes debris from the posterior side of the Achilles tendon [4-6], and the healthy RB contains highly viscous synovial fluid [8]. Its viscoelastic properties are thought to neutralize compressive stress and resist deformation or corruption during ankle motion. 
There are several limitations of this study. First, the sample size of the subjects was limited and all the subjects were young females. Second, disparity in the mechanical axis of the lower limb and the influence of gastrocnemius contraction were not evaluated. Last, the stiffness of the RB wall and the pressure of the RB fluid were not measured. Further study is needed.

\section{Conclusions}

This study showed that in healthy subjects, the thickness of the RB in the horizontal plane did not change with respect to the ankle position with or without FHL contraction.

Author Contributions: Misako Hamada, Minori Ota and Nobuhide Azuma conducted the acquisition of data; Misako Hamada and Kotaro Yamakado were drafting of manuscript and wrote the main paper. All authors discussed the study conception and design, the results and implications at all stages.

Conflicts of Interest: The authors declare no conflict of interest.

\section{References}

1. Theobald, P.; Bydder, G.; Dent, C.; Nokes, L.; Pugh, N.; Benjamin, M. The functional anatomy of Kager's fat pad in relation to retrocalcaneal problems and other hindfoot disorders. J. Anat. 2006, 208, 91-97. [CrossRef] [PubMed]

2. Benjamin, M.; Kumai, T.; Milz, S.; Boszczyk, B.M.; Boszczyk, A.A.; Ralphs, J.R. The skeletal attachment of tendons-Tendon 'entheses'. Comp. Biochem. Physiol. 2002, 133-A, 931-945. [CrossRef]

3. Rufai, A.; Ralphs, J.R.; Benjamin, M. Structure and histopathology of the insertional region of the human Achilles tendon. J. Orthop. Res. 1995, 13, 585-593. [CrossRef] [PubMed]

4. Benjamin, M.; McGonagle, D. The enthesis organ concept and its relevance to the spondyloarthropathies. Adv. Exp. Med. Biol. 2009, 649, 57-70. [PubMed]

5. Kumai, T.; Takakura, Y.; Rufai, A.; Milz, S.; Benjamin, M. The functional anatomy of the human anterior talofibular ligament in relation to ankle sprains. J. Anat. 2002, 200, 457-465. [CrossRef] [PubMed]

6. Shaw, H.M.; Benjamin, M. Structure-function relationships of enthesis in relation to mechanical load and exercise. Scand. J. Mad. Sci. Sports 2007, 17, 303-315. [CrossRef] [PubMed]

7. Johannes, I.; Somford, M.P.; Hoornenborg, D.; van Dijk, C.N. Eponyms of the Kager Triangle. J. Bone Jt. Surg. Am. 2012, 94. [CrossRef]

8. Canoso, J.J.; Liu, N.; Traill, M.R. Physiology of the retrocalcaneal bursa. Ann. Rheum. Dis. 1988, 47, 910-912. [CrossRef] [PubMed]

(C) 2016 by the authors; licensee MDPI, Basel, Switzerland. This article is an open access article distributed under the terms and conditions of the Creative Commons Attribution (CC-BY) license (http://creativecommons.org/licenses/by/4.0/). 